\title{
Reanimation of the Paralyzed Human Larynx with an Implantable Electrical Stimulation Device
}

\author{
David L. Zealear, Cheryl R. Billante, Mark S. Courey, James L. Netterville, Randal C. Paniello, \\ Ira Sanders, Garrett D. Herzon, George S. Goding, Wolf Mann, Hasse Ejnell, \\ Aifons M.M.C. Habets, Roy Testerman, Paul Van de Heyning
}

\author{
Department of Otolaryngology \\ Vanderbilt University \\ david.zealear@vanderbilt.edu
}

\begin{abstract}
Electrical stimulation of the posterior cricoarytenoid (PCA) muscle, when paced with inspiration, offers a physiologic approach to restore ventilation in bilateral laryngeal paralysis without any of the disadvantages associated with conventional treatment. In an eighteen-month prospective study, six patients were successfully implanted with an Itrel ${ }^{\circledR}$ II stimulator (Medtronic, Inc). In post-operative sessions, stimulated vocal fold abduction, patient ventilation, and voice were assessed and compared to pre-operative values. The optimum stimulus paradigm was a 1-2 second train of 1 millisecond pulses delivered at a frequency of 30-40 hertz and amplitude of 2-7 volts. PCA stimulation produced a large dynamic abduction $(3.5$ to $7 \mathrm{~mm})$ in three patients and moderate abduction $(3 \mathrm{~mm})$ in a fourth patient. The fifth patient showed a large but delayed response of $4 \mathrm{~mm}$ to stimulation with some lateralization of the vocal fold. In the sixth patient, stimulated abduction was noted upon device implantation but lost postoperatively. All five patients with stimulated abduction postoperatively met the ventilatory criteria for decannulation, and three patients were subsequently decannulated. Chronic stimulation of the PCA muscle had no appreciable effect on voice quality. Electrical stimulation of the PCA muscle shows potential as an improved therapy for bilateral vocal fold paralysis.
\end{abstract}

\section{Introduction}

Bilateral vocal fold paralysis (BVFP) is a serious and often life-threatening clinical problem. Surgical techniques such as laser arytenoidectomy and partial cordectomy can be performed to widen the airway and relieve dyspnea in case of chronic paralysis. However, these procedures compromise voice and airway protection in order to restore ventilation through the mouth. They also ignore the long-term effects of ensuing atrophy on vocal fold mass and position. In general, the greater the cartilaginous or membranous resection associated with either technique, the greater the morbidity. A number of modifications of these two strategies have been devised in an attempt to strike a more delicate balance between improved oral ventilation and impaired voice and swallowing. However, a more conservative stance toward resection increases the probability of failed intervention and the necessity for revision surgery.

A new, more physiologic approach termed laryngeal pacing has been studied in animal models as a means to restore oral ventilation. ${ }^{1,2}$ Pacing involves functional neuromuscular stimulation (FNS) of the posterior cricoarytenoid (PCA) muscles during the inspiratory phase of respiration to abduct the vocal folds. During the expiratory phase, the vocal folds passively relax to the midline to allow for normal voicing and airway protection.

The results of the animal studies provided a strong basis for a worldwide, multi-institutional FDA study sponsored by Medtronic, Inc. using an implantable laryngeal stimulator. Six patients were successfully implanted; a seventh was explanted due to infection. Preliminary results from four of the patients have been previously presented or published. ${ }^{3-5}$ The present manuscript will summarize the results across all subjects and discuss the merit of this new treatment modality.

\section{Materials and Methods}

\section{Patient History}

Six female and one male patient received an implant. The mean age of the seven patients was 60 years, with an age range from 33 to 77 years. The etiology of bilateral paralysis varied, with six patients injured during thyroidectomy and one patient presenting idiopathically. All patients had been tracheotomized in conjunction with their injury.

\section{Evaluation of Laryngeal Function}

The innervation status of laryngeal muscles was assessed in the awake patient by means of percutaneous needle electromyography. The patient was then anesthetized and the arytenoid joint palpated during direct laryngoscopy to rule out arytenoid fixation as the cause of vocal fold immobility. Device Implantation

The Itrel ${ }^{\mathbb{R}}$ II is comprised of an implantable pulse generator (IPG), a lead, and a lead tip or electrode. The electrode has multipolar contacts or channels for transmitting stimulation to a laryngeal muscle (see figure 1). After the cricothyroid joint was surgically exposed, the larynx was rotated to access the PCA muscle. An electrode containing two to four channels was inserted between the muscle and the cricoid cartilage (figure 2). The electrode lead was tunneled subcutaneously to a second incision made below the clavicle. The lead was connected to the IPG case, which contained a pulse generating circuit, long-life battery, and telemetry technology for programming the stimulation. The device as it presently exists does not have an inspiratory sensor, and is not synchronized with patient inspiratory effort. It can be programmed to stimulate at regular intervals to provide a means for air exchange, but the patient must adjust his or her respiratory rate to that of the stimulus cycle.

Postoperative Evaluation

During monthly post-operative sessions, stimulated 
vocal fold abduction, patient ventilation, and voice were assessed and compared to pre-operative values. To measure vocal fold abduction, a rigid 70-degree endoscope or flexible nasopharyngoscope was positioned to directly visualize the vocal folds. The distance between the vocal folds was measured at rest and at peak abduction during stimulation of the PCA muscle. Vocal fold width was used as the reference for measurement. The width was found to be $3 \mathrm{~mm}$ measured with a laser ruler attached to the end of the endoscope shaft.

Patient ventilation was assessed spirometrically. For patients with BVFP, the degree of glottal resistance to inspiratory flow was the primary concern. Therefore, the peak inspiratory flow (PIF) value was considered the critical indicator of this resistance. With the mouth and nose occluded, PIF measured through the trach site indexed the patient's existing level of ventilation. The tracheotomy was then occluded, and PIF measured through the mouth with the device on to determine if the device could restore ventilation through the mouth sufficient to bypass need for a tracheotomy. PIF was measured through the mouth with the device off to ascertain whether ventilation through a passive airway was at a level necessary to sustain life in the event of device failure.

Eventual decannulation required that a patient meet two criteria. The first criterion dictated that the airflow (PIF) through the mouth equalled that through the trach, or alternatively was greater than $1.5 \mathrm{1} / \mathrm{sec}$. Based on previous clinical measures of PIF through the tracheotomy, $1.51 / \mathrm{sec}$ was deemed a conservative level of airflow that would support ambulation. The second criterion for decannulation determined whether the measures of stimulated abduction and airflow provided sufficient ventilation for the patient to maintain the same activity level prior to pacing. Under physician supervision, the patient occluded the tracheotomy and kept it closed continuously for a period of two months.

Perceptual measures of voice quality were obtained using the GRBAS scale. ${ }^{6}$ A number 0 (none) to 3 (severe) was assigned to several descriptors of quality including grade, roughness, breathiness, asthenicity, and strain. ${ }^{5}$

Statistical analysis of the pre to postoperative changes in vocal fold abduction $(\mathrm{mm})$, mouth ventilation (PIF), and voice quality (GRBAS score) was performed by means of a student-t test or nonparametric test (i.e. GRBAS).

\section{Results}

In the four patients tested, EMG motor unit activity was present in the PCA and thyroarytenoid (TA) muscles during voluntary effort. Recordings showed inappropriate firing patterns indicative of synkinetic reinnervation.

Respiratory, endoscopic and voice data are shown in

Table 1. The optimum stimulus paradigm for vocal fold abduction was a 1-2 second train of 1 millisecond pulses delivered at a frequency of 30-40 pulses per second (pps) and amplitude of 2-7 volts. ${ }^{7}$ One to two seconds of stimulated abduction allowed sufficient air exchange with each breath. Video stillframes of the glottis were used to measure the distance between the vocal folds at rest and during stimulation. Measurements were taken on the vocal process at the site of maximum opening. As seen in Table 1, stimulated abduction significantly increased the magnitude of glottal opening in patients \#1-5 from preoperative levels $(\mathrm{p}<.0008)$. Stimulated glottal opening was large in patients \#1, \#3 and \#4 $(3.5-7 \mathrm{~mm})$ and moderate in patient $\# 2(3 \mathrm{~mm})$. In patient $\# 5$, stimulation also produced a large abduction of $4 \mathrm{~mm}$, but the response was delayed in time.

As mentioned previously, the principal parameter used to characterize ventilatory status was PIF. Patients \#1-5 had PIFs greater than .4 liters per second when breathing through the mouth with the device off. This level was deemed sufficient to sustain life in case of device failure in the eventually decannulated patient. As expected, the PIF values through the mouth significantly increased in each patient when the laryngeal pacemaker was activated $(p<.0069)$. Furthermore, the magnitude of PIF was generally correlated with the degree of stimulated abduction. As shown in Table 1 under "postop", the four patients with large stimulated abduction (\#1, \#3, \#4, \#5) demonstrated the greatest PIF values through the mouth with the device on. The remaining patient (\#2) had a lower PIF value. Nonetheless, all five patients had PIF values surpassing that through the trach site or greater than $1.5 \mathrm{l} / \mathrm{sec}$, and therefore all five fulfilled the first criterion for decannulation. The second criterion dictated that the patient could function in day to day activity with tracheostomy occluded and relying solely on the device. This criterion avoided a clinical decision based solely on the measure of PIF. All five patients also fulfilled this requirement. Patients $\# 1,4$ and 5 were subsequently decannulated. One patient (\#3) expired from cancer recurrence before decannulation could be performed. In the final patient (\#2), the decision to decannulate was deferred because only nominal values of PIF were recorded from the trach or mouth.

Continual stimulation of the PCA muscle over the entire course of study had null effect on voice (p>.39). Two of the patients $(\# 4, \# 5)$ became slightly breathy following implant surgery, but no subsequent change was noted in voice quality following activation of the pacer. In all five patients, perceptual judgments of voice quality found no change that could be attributed to longterm stimulation. All testing was performed with the device off to evaluate the cumulative effect of electrical stimulation. Further, a more detailed voice analysis of patients \#1 and 2 showed no significant change in acoustic measures of voice, including dynamic frequency and intensity range. Maximum phonation times were consistent across pre- and post-operative sessions. Airflow rates during voicing did not change with stimulation of the PCA muscle over the course of the investigation.

During the study, several technological limitations were observed with the pacemaker device in both its design and hardware. First, while the lack of effect of chronic stimulation on voice was well documented, there was concern that stimulated abduction unsynchronized with respiration might interrupt voicing in conversational speech. In four of five patients, endoscopic examination of the larynx revealed that the patient could override stimulated glottal opening with no perceived change in voice quality. However, the patient with delayed vocal fold opening (i.e. \#5) was unable to override the effects of stimulation. Voice quality was mildly breathy and asthenic when the device was continuously active. There was also concern that stimulated abduction unsynchronized with respiration might lead to aspiration during swallowing. Atlhough fluoroscopic examination of barium swallow was not performed to assess deglutition, there were no reports of aspiration during swallowing in any of the patients. Further, none of the participating physicians reported patient symptoms of silent aspiration as indicated by spiking temperature, pulmonary congestion or pneumonia. 
Two problems were encountered with the device hardware. When using bipolar stimulation, the electrode proved susceptible to electrochemical corrosion at the anode (positive pole). Deterioration could be largely attributed to charge accumulation from the relatively long pulse duration required for this application. If stimulation was shifted to a monopolar configuration, the IPG case could serve as a suitable anode alternative. Given the large surface area of the IPG, anode corrosion was then circumvented. Over the longterm, adoption of a monopolar format avoided corrosion and loss of response in two patients (\#3, \#5 Table 1) and compensated for corrosion with regain of response in one patient (\#1). However, monopolar stimulation was ineffective in reestablishing stimulated abduction in the other two patients $(\# 2, \# 4)$. The second hardware limitation related to the size and spacing of electrode channels or contacts. The contacts were too large and too widely spaced for optimum stimulation of the tiny PCA muscle. This electrode design required a moderate degree of accuracy in electrode placement, otherwise pca activation may fail or excessive current spread may occur during application of the stimulus to the PCA. In this regard, suboptimal electrode placement was likely responsible for failed abduction in patient \#6. Further, it was discovered that the current had spread beyond the PCA borders in patient \#1, leading to activation of nerve fibers that had reinnervated adductor muscles. The recruitment of the adductors antagonized stimulated abduction in this patient. Periodic injection of botulinum toxin was necessary to block antagonistic activity of the TA muscle and allow uncontested abduction. Figure 3 shows an endoscopic view of the paralyzed larynx before implantation (3A) and following reanimation with the Itrel ${ }^{\circledR}$ stimulator in patient $\# 1$ treated with Botox (3B).

Patient status has also been followed until the present, as long as six years after pacemaker implantation. The long-term outcome for each patient is shown in the final row of table 1. Normal activity levels were maintained in patients \#1-5. Anode corrosion and device failure eventually occurred in two of the patients (\#2 and \#4) at six and thirty months respectively. Stimulated abduction became inadequate in these two patients. The remaining three patients (\#1, \#3, \#5) retained their therapeutic level of abduction, ventilation, and voice over the long-term.

\section{Discussion}

This study represents the first successful clinical application of functional electrical stimulation to reanimate paralyzed muscles of the head and neck. The findings demonstrate the merit of laryngeal pacing as a new treatment approach. Electrical pacing restored vocal fold movement with a peak glottal opening as large as $7 \mathrm{~mm}$. Stimulated vocal fold abduction on only one side was sufficient to restore airflow through the mouth. An inspiratory airflow of greater than 1.5 liters per second allowed a patient to maintain his or her normal everyday activity, which proved adequate for decannulation. The fact that chronic electrical pacing caused no disturbance of voice quality further validated the physiologic nature of this treatment. Improved ventilation with null effect on voice attest to the advantage of laryngeal pacing over conventional surgical therapies for BVFP.

\section{References}

[1] Zealear DL, Dedo HH. Control of paralyzed axial muscles by electrical stimulation. Acta Otolaryngol (Stockh) 1977;83:514-27.

[2] Obert PM, Young KA, Tobey DN. Use of direct posterior cricoarytenoid stimulation in laryngeal paralysis. Arch Otolaryngol 1984;110:88-92.

[3] Van de Heyning P, Sanders I, Goding GS, Herzon GD. Human trials of an implanted laryngeal pacer for bilateral vocal fold parlaysis (abstract). Otolaryngol Head Neck Surg 1996;115(2):P49.

[4] Zealear DL, Billante CR, Courey MS, Sant'Anna GD, Netterville JL. Electrically stimulated glottal opening combined with adductor muscle botox blockade restores both ventilation and voice in a patient with bilateral laryngeal paralysis. Ann Otol Rhinol Laryngol 2002;111(6):500-6.

[5] Billante CR, Zealear DL, Courey MS, Netterville JL. Effect of chronic electrical stimulation of laryngeal muscle on voice. Ann Otol Rhinol Laryngol 2002;111:328-32.

[6] Hirano M. Clinical examination of voice. New York: Springer-Verlag Wien, 1981.

[7] Zealear DL, Swelstad MR, Sant'Anna GD, Bannister R, Billante CR, Rodriguez RJ, Garren KC, Billante MJ, Champney MS. Determination of the optimal conditions for laryngeal pacing with the itrel II implantable stimulator. Otolaryngol Head Neck Surg. 2001:125(3);183- 


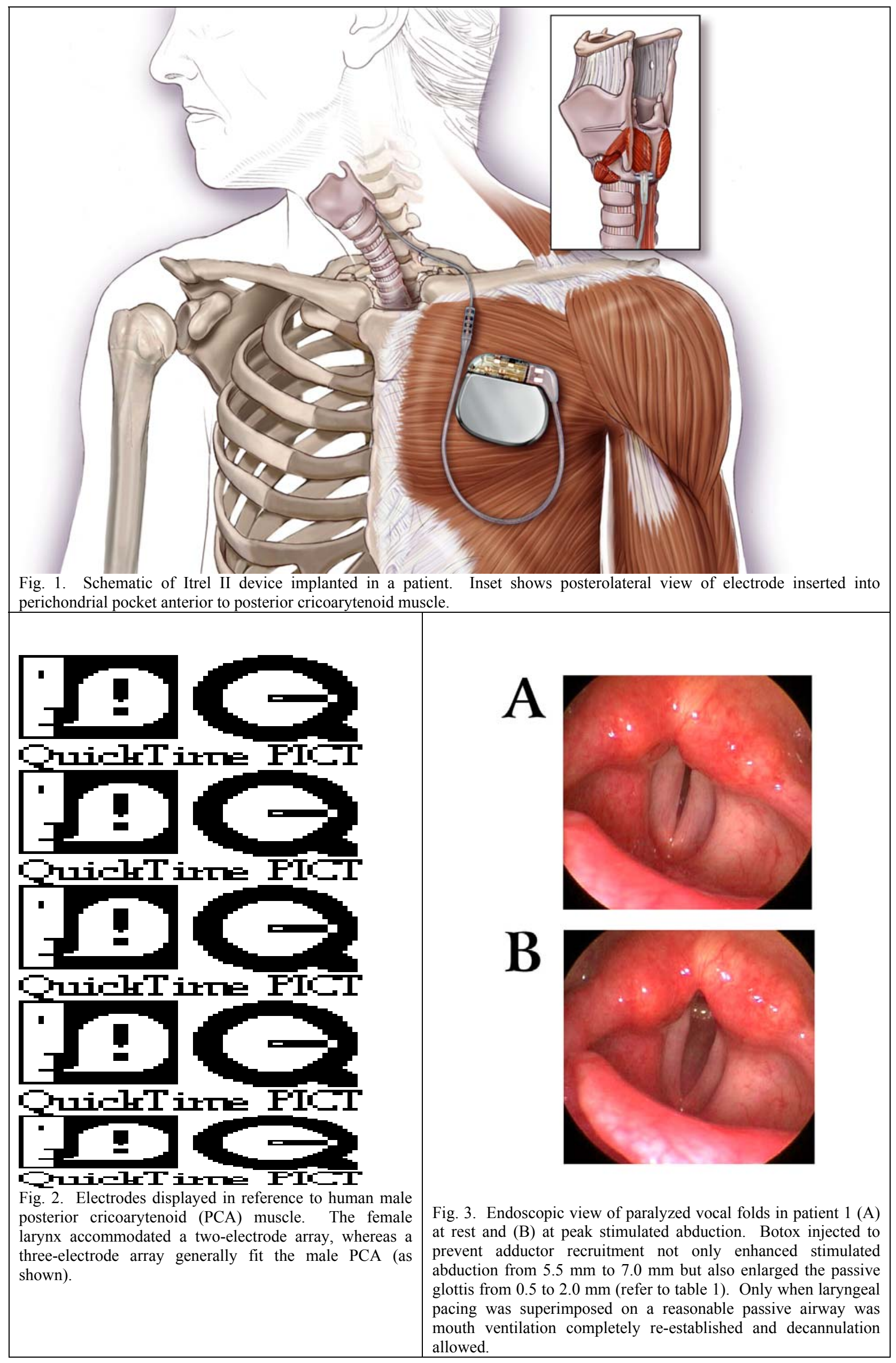


TABLE I.

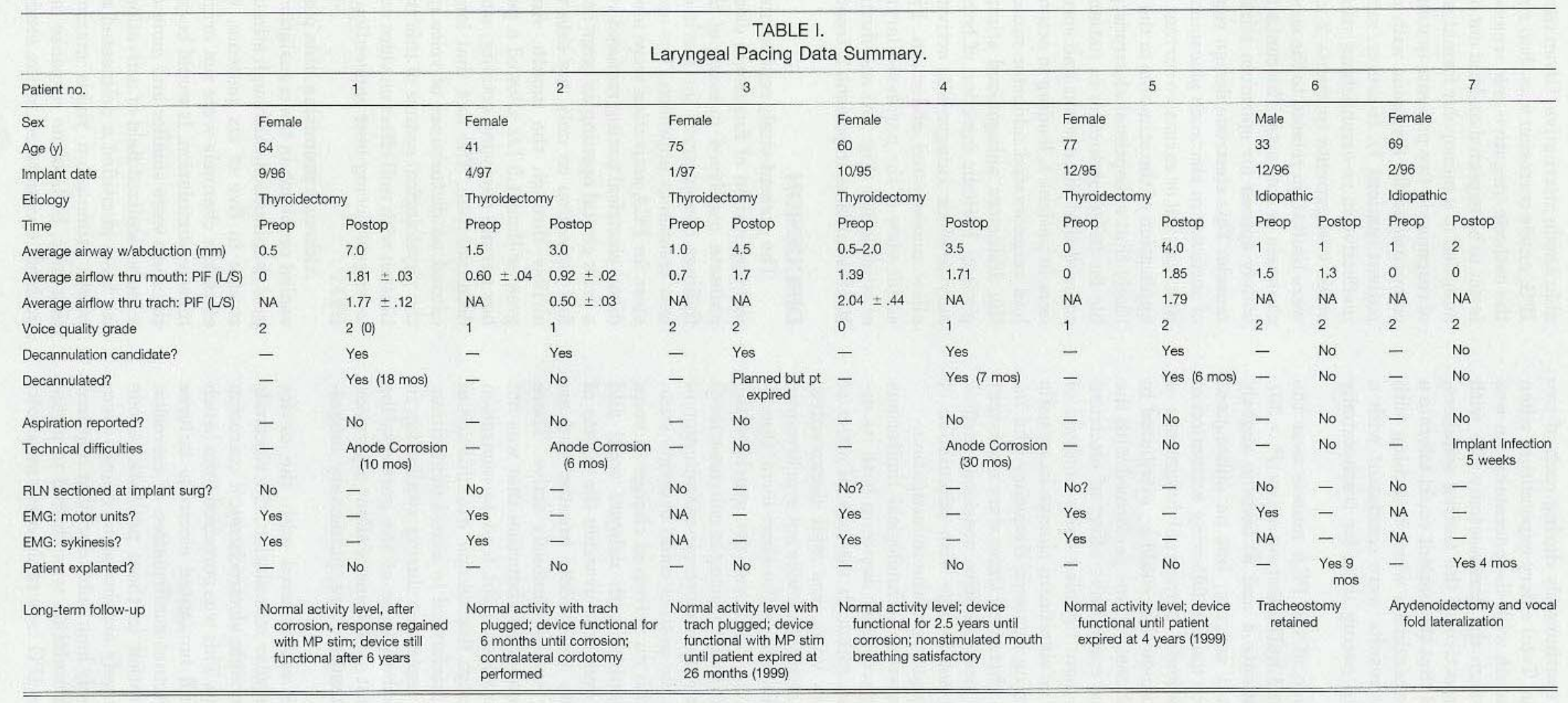

